\title{
EI sistema de las Creative Commons
}

\author{
Por Marco Marandola
}

\begin{abstract}
Resumen: El movimiento Creative Commons representa la vertiente jurídica de los elementos que componen el copyleft. Las licencias se basan en algunos puntos fundamentales: todo titular de la copia de una obra protegida por la licencia puede utilizarla sin límites; distribuir o redistribuir tantas copias como desee y modificarla del modo que desee, siempre que se juzgue conveniente. Existen tres esquemas generales de licencias: las common deed, las legal code y las digital code. Los Creative Commons son el primer intento de proveer de un marco jurídicamente válido al acceso abierto.

Palabras clave: Creative Commons, Open access, Copyleft, Licencias, Internet, Derecho de autor, Derechos de explotación.
\end{abstract}

\section{Title: Creative Commons scheme}

Abstract: Creative Commons is the first attempt to provide a valid legal framework for open access. This text analyses the beginnings of the movement and the different types of licences that have been prepared, as well as offering considerations regarding Creative Commons applications.

Keywords: Creative Commons, Open Access, Copyleft, Licences, Internet, Copyright, Economic rights

EL MOVIMIENTO CREATIVE COMMONS representa la vertiente jurídica de los diversos elementos que componen el copyleft. Se trata de la redacción de las licencias y los contratos que determinan en qué condiciones se puede utilizar la obra, o mejor dicho, las obras, ya que se prevén licencias para varios tipos de soporte. Las licencias se redactan (o se redactarán) en diversas lenguas para facilitar su comprensión.

Uno de los objetivos principales que se persiguen es: "los creadores o los autores pueden querer que se aplique el copyleft a su obra, y gracias ello esperan generar las condiciones más favorables para que una gran cantidad de personas se sientan animadas a contribuir con mejoras y elaboraciones de dicha obra, en un proceso continuo". http://www.creativecommons.org

James Boyle (experto en "ciberleyes"), Michael Carroll, Lawrence Lessing, Hal Abelson

Este artículo es parte del libro:

Marandola, Marco. ¿Un nuevo derecho de autor? Introducción a copyleft, acceso abierto y Creative Commons. Barcelona: Derecho de autor SL, 2005. Isbn 8460960307. http://www.derechoycultura.com (profesor del Instituto de Tecnología de Massachusetts), Eric Saltzman y Eric Eldred (editor web de dominio público) fundaron Creative Commons en 2001 financiados por la Facultad de Derecho de la Universidad de Stanford y por la Sociedad del Dominio Público.

El primer proyecto fue en diciembre de 2002, cuando se desarrolló una serie de licencias para web tomando como inspiración (en parte) la Free Software Foundation's GNU General Public Licence (GNU GPL). Danilo Moi escribe lo siguiente en el número $\mathrm{V}$ de Diritto e cultura: "Creative Commons, la revolución pacífica a través del derecho". Sin embargo, el salto cualitativo del proyecto se da 
con la institución de los Internacional Commons.

En verano de 2003, Brasil, Finlandia y Japón se comprometieron (a través de la Fundación Getulio Vargas Law School de Río de Janeiro, el Instituto de Tecnologías de la Información de Helsinki y el Glocom, respectivamente) a adoptar y promocionar el proyecto en sus territorios. La idea de base es desarrollar las licencias en los países en función de la compatibilidad con los sistemas jurídicos locales.

\section{«Las licencias \\ Creative Commons hacen referencia a una amplia esfera de obras protegidas, pero no a los programas informáticos»}

En el transcurso de este último año se ha producido una veintena de adhesiones, todas a través de prestigiosas universidades: el proyecto se ha convertido ya en un fenómeno de escala internacional.

La conciencia de la exigencia de un apoyo institucional es requisito fundamental para el objetivo básico: el reconocimiento legal de las licencias es para Creative Commons un factor evidente e inequívoco. Este es precisamente uno de los puntos fuertes del proyecto, $\mathrm{y}$ quizás el principal, y cabe detenerse en él.

Las licencias Creative Commons hacen referencia a una amplia esfera de obras protegidas, pero no a los programas informáticos.

\section{Las bases de las licencias}

Todo titular de una copia de una obra protegida por la licencia puede:

_Utilizarla sin límites.
- Distribuir o redistribuir tantas copias como desee.

-Modificarla siempre que lo considere conveniente.

De todos modos, falta aún un elemento básico: que la obra derivada siga estando bajo copyleft. ¿Qué significa esto? Una obra que se modifica (sustancialmente) pasa a ser otra nueva sobre la cual nacen nuevos derechos a favor del autor. Sin embargo, para poder llevar a cabo la modificación es preciso contar con el permiso de su primer autor. Por lo tanto, quien transforma una obra que tiene copyleft, porque la licencia así lo permite, obtiene una serie de derechos nuevos sobre el nuevo producto que puede oponer a cualquier otra persona (copyright). Para evitar este absurdo, la licencia permite modificar una obra con copyleft únicamente si quien lo hace se compromete a mantenerla bajo este criterio, y así sucesivamente.

Otros posibles elementos de una licencia Creative Commons son:

-Que las condiciones no puedan ser revocadas.

-Asegurarse de que la obra y sus versiones derivadas estén siempre en un soporte que permita su modificación.

- Garantizar que la obra original quede documentada, así como sus versiones modificadas.

\section{Las licencias}

Las licencias pueden ser diferentes en función de los países, de conformidad con las leyes nacionales de cada uno en materia de propiedad intelectual.

Toda licencia se materializa en tres esquemas generales:

-Common deed: versión "humanamente comprensible" o escritura común.
- Legal code: licencias con verdaderas cláusulas jurídicas.

—Digital code: ayudan a los buscadores a identificarlas.

\subsection{España}

Las licencias common deed son las siguientes:

\section{Atribución}

Se es libre de:

—copiar, distribuir y comunicar públicamente la obra;

- hacer obras derivadas;

-hacer uso comercial;

Bajo las condiciones siguientes:

—reconocimiento y cita del autor original;

- al reutilizar o distribuir la obra hay que dejar bien claros los términos de la licencia.

\section{Reconocimiento, compartir igual}

Se es libre de:

- copiar, distribuir y comunicar públicamente la obra;

—hacer obras derivadas;

-hacer un uso comercial;

Bajo las condiciones siguientes:

- reconocimiento y cita del autor original;

- compartir bajo la misma licencia; si se altera o transforma la obra, o se genera otra obra derivada, ésta sólo puede distribuirse bajo una licencia idéntica.

\section{Reconocimiento, sin obra de-} rivada

Se es libre de:

- copiar, distribuir y comunicar públicamente la obra;

—hacer un uso comercial de ella;

Bajo las condiciones siguientes:

-reconocimiento y cita del autor original; 


\section{SISTIMIA SABINI}

\section{Soluciones integrales para la Automatización de Bibliotecas y Centros de Documentación}

Desarrollado integramente en castellano con más de 15 años de experiencia en España y Latinoamérica, atiende las necesidades de todo tipo y tamaño de bibliotecas y centros de información

\section{MÓdULOS:}

- Adquisiciones

Trámite de pedidos

Gestión de gastos y proveedores

- Catalogación

Catalogación de todos los materiales biblográticos Integración de información bibliográfica

- Terminología

Ficheros de autoridades Tesauro multilingūe

- Consultas

Lenguaje de Comandos

Acoeso publico en linea (CAPEL)

Acceso a través de WWW

Protocolo Z39.50

- Circulación

Gestión de préstamos

Fichero de usuarios

- Estadísticas

De proceso y circulación

\section{Otros servicios:}

Instalación y soporte técnico del sistema SABINI

Asesoría en Automatización de Bibliotecas y Centros de Documentación

Procesamiento de material bibliográfico

Instalación de catálogos en Internet

Cr Amor de Dios, $\pi^{*} 1$

Tell: +3491429255 ?

Fax +34914298610

28014 MADRD

e-mall: sabivi6sabinicom

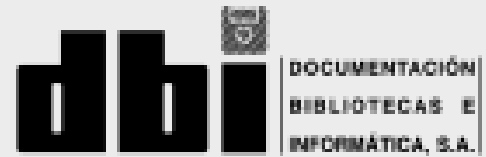

Av. Diez Cansoco 236 of. 602 Lma 18

Telotax: [511) 446-0315 e-mail: anbinissorma campe 
— sin obras derivadas; no se puede alterar, transformar o generar una obra derivada a partir de la original.

\section{cial}

Reconocimiento, no-comer-

Se es libre de:

- copiar, distribuir y comunicar públicamente la obra;

—hacer obras derivadas;

Bajo las condiciones siguientes:

—reconocimiento y cita del autor original;

—no se puede utilizar la obra para fines comerciales.

\section{Reconocimiento,no-comer-} cial, compartir igual

Se es libre de:

- copiar, distribuir y comunicar públicamente la obra;

—hacer obras derivadas;

Bajo las condiciones siguientes:

-reconocimiento y cita del autor original;

—no se puede utilizar la obra para fines comerciales;

- compartir bajo la misma licencia.

\section{Reconocimiento, no-comer- cial, sin obra derivada}

Se es libre de:

- copiar, distribuir y comunicar públicamente la obra;

Bajo las condiciones siguientes:

-reconocimiento y cita del autor original;

—no se puede utilizar la obra para fines comerciales;

— sin obras derivadas; no se puede alterar, transformar o generar una obra derivada a partir de la original.

Una cláusula es común a todas las licencias: al reutilizar o distri- buir la obra se tienen que dejar bien claros los términos de su licencia.

Hay que tener en cuenta que Creative Commons no es un bufete de abogados y no ofrece servicios legales. Sólo identifica diferentes tipos de no-protección bajo el copyleft. La distribución, la muestra, o el enlace de este resumen no crean ningún vínculo abogadocliente.

Hay licencias para diferentes medios: texto, imagen, vídeo, música.

\section{Algunas consideraciones}

Los sistemas de "derecho de autor", como el de España, y los de "copyright" propiamente dicho, como el de los EUA, no se limitan a meras diferencias entre ambos sistemas legales y algunas son notables.

En la tradición anglosajona del Common Law por la que se rige EUA, el sistema se identifica por pocas leyes, muchos contratos y una infinidad de sentencias que sientan el precedente que para nosotros tiene el valor de ley. En los sistemas mediterráneos, el legislador siempre está presente, con numerosas leyes para desempeñar una función supra partes.

Estas diferencias son patentes, por ejemplo, en la amplia tutela que reconoce el código civil a las partes en los contratos o licencias, en los que ciertas cláusulas o condiciones no pueden superarse nunca, ni siquiera de mutuo acuerdo entre las partes.

La licencia Creative Commons, por lo tanto, se adapta bien a un sistema legal como el anglosajón, puesto que las partes regulan casi con absoluta libertad la relación jurídica que se establece entre ellas. En nuestro sistema de Civil Law, dicho esquema contractual topa con los límites de las ordenanzas legales españolas.

Sin embargo surge una nueva duda respecto a la licencia que permite las obras derivadas: según la misma el autor autoriza a priori a cualquier persona a modificar su obra, crear una nueva a partir de ella y por lo tanto contemplar una nueva paternidad sobre el original.

Según la Ley de propiedad intelectual española, los derechos morales son intransmisibles, y por tanto no pueden ser cedidos, ni siquiera con el consentimiento del autor.

\section{«La licencia permite modificar una obra con copyleft únicamente si quien lo hace se compromete a mantenerse bajo este criterio, y así sucesivamente»}

Otra consideración es la extensión y la complejidad de la licencia en su versión íntegra, lo que coloquialmente ha venido a llamarse "para abogados". Se trata de una edición considerablemente complicada que todavía no se adapta a la perfección a nuestro sistema jurídico. Siguen existiendo algunos problemas, como por ejemplo el lugar de celebración del contrato, la validez del mismo y las consecuencias y sanciones en caso de incumplimiento.

Un último comentario: a pesar de la exactitud de la redacción, y como se acaba de apuntar, las licencias Creative Commons no consiguen adaptarse perfectamente a todas las realidades y a todas las necesidades.

El autor se encontró con uno de esos casos. Al terminar de escribir la versión italiana del libro "Un nuovo diritto d'autore, copyleft, open access e creative commons", 
había conseguido convencer al editor de que permitiese la difusión de la obra en formato digital, ya que defiende los ideales del acceso abierto y de Creative Commons, y por lo tanto le parecía que eso era su deber.

El editor aceptó con dos condiciones: que la obra no pudiese imprimirse y que si con las ventas no se cubriesen, como mínimo, los gastos de impresión, el autor tendría que sufragarlos personalmente.

Cierto es que ninguna licencia Creative Commons italiana preveía en aquel momento la posibilidad de que fuera difundida en dos soportes (digital y papel). Por lo tanto, la versión electrónica se puso libremente a disposición de todo el mundo, acompañada de esta sencilla fórmula:

(C) Algunos derechos reservados. El autor y el editor consienten la libre difusión de la obra en formato electrónico, pero queda prohibida su impresión, su modificación y su difusión comercial o lucrativa.

En el fondo eso pareció más sencillo y más simple, y también más útil en su objetivo, que cualquier licencia Creative Commons.

\section{Conclusiones}

Las Creative Commons representan un paso fundamental en una nueva gestión de las obras, y del

conocimiento, ya sea dentro del acceso abierto o del copyright.

La experiencia bien establecida ya en algunos países (por lo general, anglosajones) y sobre ciertos tipos de obras (electrónicas en el ámbito universitario) ha permitido el desarrollo del mismo movimiento open access en sí.

Sin embargo, no puede decirse que en todos los casos haya sido fructífero el experimento de brindar a algunos institutos de diversas partes del mundo la posibilidad de traducir, e incluso de adaptar las licencias a las diversas legislaciones nacionales. Las licencias siguen siendo muy "jóvenes", y es normal que puedan evolucionar para adaptarse a las peculiaridades de los sistemas jurídicos, sin tener que ser necesariamente uniformes entre sí.

\section{"Las licencias siguen siendo muy 'jóvenes', $y$ es normal que puedan evolucionar para adaptarse a las diversas necesidades de los distintos sistemas jurídicos, sin tener que ser necesariamente uniformes entre sí»}

En realidad, la licencia (o contrato), además de la ley nacional, sirve para proteger (en parte) la obra puesta a disposición de la comunidad mundial de estudiosos, pero no resuelve, a nuestro entender, todos los problemas de los diferentes tipos de usos, ni todos los tipos de obras.

Además, dada la novedad de la licencia, la comunidad de los $\mathrm{Cre}$ ative Commons difícilmente se ha convertido en portadora, a nivel mundial, de lobbies influyentes o de propuestas de modificación de la ley nacional de derecho de autor. En ciertos países algunos commoners han intentado lanzar proposiciones de leyes que apoyasen la adopción de software libre, o el libre acceso a las obras producidas o financiadas gracias a las subvenciones públicas, pero estas iniciativas han constituido más bien casos aislados que un movimiento coordinado.

Debe reconocerse el indudable mérito de los esquemas contractuales de Creative Commons como primer intento de proveer de un marco jurídicamente válido al acceso abierto. No obstante, personalmente creemos que el potencial de la adopción de un esquema contractual válido y eficaz diverso en función de los diferentes tipos de obras y de usos (académico, privado, universitario, cultural, comercial) todavía tiene que centrarse plenamente.

Marco Marandola, Asesor jurídico en derecho de autor, IFLA CLM. marandol@tiscali.es

http://www.derechoycultura.com

El profesional de la información está abierto a todos los bibliotecarios, documentalistas y otros profesionales de la información, así como a las empresas y organizaciones del sector para que puedan exponer sus noticias, productos, servicios, experiencias y opiniones.

Dirigir todas las colaboraciones para publicar a:

El profesional de la información

Apartado 32.280

08080 Barcelona

epi@elprofesionaldelainformacion.com 